\title{
Modeling of A Bearing Test Bench and Analysis of Defect Bearing Dynamics in Modelica
}

\author{
Diwang Ruan ${ }^{1} \quad$ Zhirou Li $^{2} \quad$ Clemens Gühmann ${ }^{1}$ \\ ${ }^{1}$ Chair of Electronic Measurement and Diagnostic Technology, TU Berlin, Germany \\ diwang.ruan@campus.tu-berlin.de, clemens . guehmann@tu-berlin.de \\ ${ }^{2}$ School of Electronic Engineering and Computer Science, TU Berlin, Germany, lizhirou0423@hotmail.com
}

\begin{abstract}
In data-driven bearing fault diagnosis, sufficient fault data is fundamental for algorithm training and validation, however, in most industry applications, only very few fault measurements can be provided, which brings bearing dynamics model as an alternative to produce bearing response under defects. In this paper, a Modelica model for the whole bearing test rig was built, including test bearing, driving motor and hydraulic loading system. For the test bearing, a 5 degree-of-freedom (5-DoF) model was proposed to identify the normal bearing dynamics, and a fault model was employed to characterize the defect position, defect size, defect shape and multiple defects. Theory and process to implement the virtual bearing test bench in Modelica were detailed, and 3 cases were conducted to validate the effectiveness of the proposed model.
\end{abstract}

Keywords: Bearing Diagnosis, Fault Modeling, Modelica, Bearing Test Bench

\section{Introduction}

Dynamics simulation under defect is essential for bearing fault diagnostics. However, traditional research method based on experiments is of high cost and low efficiency since it requires a real test rig and the defect needs to be generated artificially. Furthermore, out of safety consideration, experiment-based research usually runs under only some specific working conditions and defect sizes, which restricts the exploration of bearing dynamics under extreme conditions and fault specifications. To bridge the gap, this paper proposes a virtual bearing test bench in Modelica to serve as a general platform for fault bearing dynamics simulation.

To date, the methods for fault bearing dynamics simulation can be classified into 2 categories, namely mechanism-based models and signal-based models. Cui et al. (Cui, X. Chen, and S. Chen (2015)) built a 5-DoF model to characterize bearing's dynamic behavior. Besides, a defect model was also established to deal with defect position, defect shape and defect size (Liu, Shao, and Lim (2012)). Whereas, other researchers investigated the fault bearing dynamics response from the perspective of signal analysis. The first model identifying the amplitude spectrum of bearing with a single defect on the in- ner race was proposed by McFadden in 1983 (McFadden and Smith (1984)). In 2000, slight random variations were further incorporated into the impulse responses to resemble actual vibration signals caused by bearing faults (Ho and R. Randall (2000)). After that, Cong et al. (Cong et al. (2013)) put forward a new fault signal model for bearing based on the combination of decaying oscillation fault signal model and rotor dynamic response influence, especially, the defect load was divided into alternate load and determinate load.

For real test bench, dynamics from driving and loading systems also affect test bearing response. Nevertheless, to our exhausted knowledge, nearly all published papers on bearing fault modeling only focus on the bearing and just set speed and load as constants, without considering the dynamics from driving motor and loading actuator, which leads to the goal to build a whole bearing test bench in this paper.

The remainder of this paper is organized as follows. Section 2 details the modeling theory of bearing test bench, including 5-DoF bearing dynamics model, bearing defect model, driving and loading system model. Section 3 outlines the model structure in Modelica and demonstrates how to use this virtual test bench to simulate bearing with specific defects. Section 4 concludes this paper.

\section{Modeling in Modelica}

\subsection{Test Bearing}

The ball bearing is composed of outer ring, inner ring, cage and rolling elements. A normal bearing achieves dynamic balance in a stable operating condition, while a series of impulses will be generated once there is a defect between the contact surfaces. In the following, a 5-DoF dynamics model and a defect model will be introduced.

\subsubsection{5-DoF Dynamics Model}

This model describes the nonlinear dynamic behavior of bearing, as shown in Figure 1. In the 5-DoF model, 4 DoF represents the horizontal and vertical direction of inner and outer rings, and 1 DoF stands for the vertical direction of a unit resonator, which is modeled as spring-mass system (Cui, X. Chen, and S. Chen (2015)).

Based on Newton's second law, the bearing dynamic 


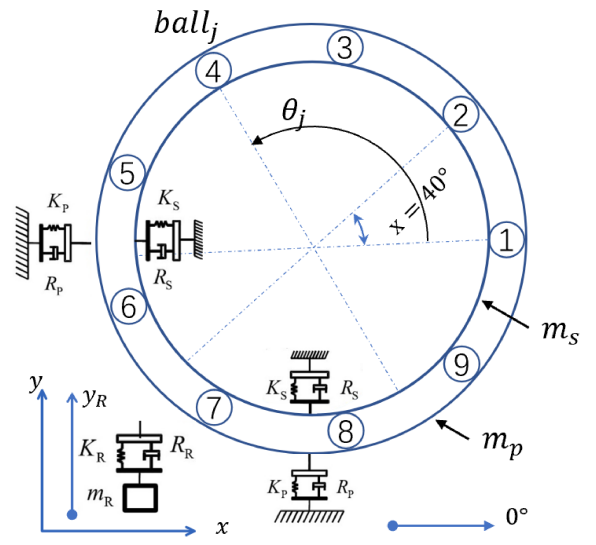

Figure 1. The 5-DoF model of bearing (Cui, X. Chen, and S. Chen (2015)).

equilibrium equations can be formulated as Equation 1 (Cui, X. Chen, and S. Chen (2015)).

$$
\begin{aligned}
& m_{s} \ddot{x_{s}}+R_{s} \dot{x_{s}}+K_{s} x_{s}+f_{x}=0, \\
& m_{s} \ddot{y_{s}}+R_{s} \dot{y_{s}}+K_{s} y_{s}+f_{y}=F_{y}-m_{s} g, \\
& m_{p} \ddot{x_{p}}+R_{p} \dot{x_{p}}+K_{p} x_{p}-f_{x}=0, \\
& m_{p} \ddot{y_{p}}+\left(R_{p}+R_{R}\right) \dot{y_{p}}+\left(K_{p}+K_{r}\right) y_{p}-R_{R} \dot{y_{b}}, \\
& -K_{R} y_{b}-f_{y}=-m_{p} g, \\
& m_{R} \ddot{y_{b}}+R_{R}\left(\dot{y_{b}}-\dot{y_{p}}\right)+K_{R}\left(y_{b}-y_{p}\right)=-m_{R} g .
\end{aligned}
$$

$f_{x}$ and $f_{y}$ are contact force at $x$ and $y$ axis respectively, $F_{y}$ is external load. The meanings and values of other variables are summarized in Table 1. According to Hertzian contact theory, the contact force between rolling element and raceways can be given by:

$$
f_{j}=K_{b} \delta_{j}^{1.5},
$$

with $j$ from 1 to $n_{b}, n_{b}$ is the number of rolling elements. $K_{b}$ stands for ball's stiffness, $\delta$ denotes deformation. The deformation of the $j^{\text {th }}$ ball, $\delta_{j}$, is determined by the displacement between the inner and outer races, the angular position $\theta_{j}$ and the total clearance $c$ caused by the oil film and assembly clearance, as:

$$
\delta_{\text {raw }_{j}}=\left(x_{s}-x_{p}\right) \cos \theta_{j}+\left(y_{s}-y_{p}\right) \sin \theta_{j}-c .
$$

The angular position of the $j^{\text {th }}$ ball can be calculated by Equation 4.

$$
\theta_{\mathrm{raw}_{j}}=\frac{2 \pi(j-1)}{n_{b}}+\omega_{c} t+\phi_{0}
$$

where $\phi_{0}$ is initial cage angular position and $\omega_{c}$ is cage angular frequency, which can be further obtained from shaft frequency $\omega_{s}$ like Equation 5.

$$
\omega_{c}=\left(1-\frac{D_{b}}{D_{p}}\right) \frac{\omega_{s}}{2},
$$

where $D_{b}$ and $D_{p}$ are the ball diameter and pitch diameter respectively.
Normally, for bearings in real applications, there exists inevitable sliding when a ball rolls on the raceways. The sliding direction depends on where the ball is located, when the ball enters into the load zone, the angular speed of the ball center is faster than that of the cage, otherwise, the ball slides backward. Consequently, when sliding considered , the angular position of each ball can be modified by Equation 6 (Cui, X. Chen, and S. Chen (2015)).

$$
\theta_{j}=\theta_{\mathrm{raw}_{j}}+\xi_{j}\left(\frac{1}{2} \text { rand }\right) \phi_{\text {slip }} .
$$

There are two constants and a sign function in Equation 6. $\phi_{\text {slip }}$ is a parameter defining the mutation percentage of average contact frequency, which is normally between 0.01 and $0.02 \mathrm{rad}$. rand is a random number with uniform distribution in the range of $[0,1]$, and the sign function $\xi_{j}$ is expressed as:

$$
\xi_{j}=\left\{\begin{array}{lr}
1, & \text { load zone } \\
-1, & \text { else }
\end{array}\right.
$$

Considering $\delta_{j}$ should be nonnegative in physics, thus, its final value is determined by:

$$
\delta_{j}=\operatorname{Max}\left(\delta_{\mathrm{raw}_{j}}, 0\right) .
$$

With the contact force of each ball obtained from Equation 2, the total contact forces in $x$ and $y$ direction can be determined with the Equations 9 and 10.

$$
\begin{aligned}
& f_{x}=\sum_{j=1}^{n_{b}} f_{j} \cos \theta_{j}, \\
& f_{y}=\sum_{j=1}^{n_{b}} f_{j} \sin \theta_{j} .
\end{aligned}
$$

\subsubsection{Defect Model}

When bearing has defects either on races or balls, an additional deformation, $\boldsymbol{\delta}_{f a u}$, will release when ball moves over the defect zone. Thus, with defect considered, deformation of the $j^{\text {th }}$ ball can be further identified as:

$$
\delta_{\text {raw }_{j}}=\left(x_{s}-x_{p}\right) \cos \theta_{j}+\left(y_{s}-y_{p}\right) \sin \theta_{j}-c-\delta_{f a u_{j}} .
$$

Once bearing deformation under defect is obtained, it can be substituted into Equations 9 and 10, where the nonlinear contact force can be calculated and further substituted into Equation 1 to get the fault bearing response. Apparently, $\boldsymbol{\delta}_{\text {fau }}$ changes with defect position, defect shape and number of defects, which will be discussed respectively in the following.

\subsubsection{Defect Position}

Firstly, four basic geometrical parameters are chosen to characterize the defect, as demonstrated in Figure 2, the defect width $B$, the defect depth $H_{d}$, the defect initial angle $\phi_{d}$ and the defect span angle $\Delta \phi_{d}$. Take a defect on the 
outer ring as an example, the relation between $\Delta \phi_{d}$ and $B$ can be expressed as:

$$
\sin \left(\frac{1}{2} \Delta \phi_{d}\right)=\frac{B}{D_{b}+D_{p}}
$$

Suppose the local defect depth is $c_{d}$, the additional defor-

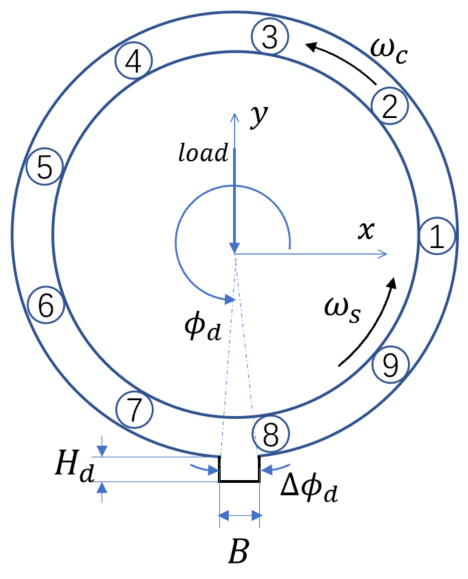

Figure 2. Size definition of defect on the outer ring.

mation $\delta_{f a u}$ generates only when a ball falls into the defect zone within $\phi_{d}$ and $\phi_{d}+\Delta \phi_{d}$, so the deformation released by defect on raceway is given by:

$$
\delta_{\text {fau }_{j}}=\left\{\begin{array}{lr}
c_{d}, & \phi_{d} \leq \theta_{j} \leq \phi_{d}+\Delta \phi_{d} \\
0, & \text { else }
\end{array}\right.
$$

The defect location on the outer ring or inner ring changes with different rules. For the outer ring, the defect is fixed at the defect initial angle $\phi_{d o}$, however, for the inner ring, the defect location changes with time when the inner ring rotates. Thus, $\phi_{d}$ in Equation 13 can be further modeled as follows.

$$
\phi_{d}= \begin{cases}\phi_{d o}, & \text { defect on the outer ring } \\ \omega_{s} t+\phi_{d i}, & \text { defect on the inner ring }\end{cases}
$$

Different from rings, when a defect happens on a rolling element, the defect spins with ball speed $\omega_{b}$ and its position $\phi_{s}$ can be obtained like:

$$
\phi_{s}=\omega_{b} t+\phi_{s_{i n i}}
$$

hereby the ball speed $\omega_{b}$ can be calculated from shaft speed as follows,

$$
\omega_{b}=\frac{\omega_{s}}{2} \frac{D_{p}}{D_{b}}\left[1-\left(\frac{D_{b}}{D_{p}} \cos \alpha\right)^{2}\right] .
$$

The defect on balls contacts the inner and outer ring periodically. Besides, the curvature radiuses of inner and outer rings are different, therefore, the same defect span angle $\Delta \phi_{d}$ produces different angular widths. The angular widths of defect on the outer ring and inner ring, $\Delta \phi_{b o}$ and $\Delta \phi_{b i}$, can be calculated by Equations 17 and 18 .

$$
\begin{aligned}
\Delta \phi_{b o} & =\Delta \phi_{d} \frac{D_{b}}{D o}, \\
\Delta \phi_{b i} & =\Delta \phi_{d} \frac{D_{b}}{D i},
\end{aligned}
$$

with $D_{o}$ and $D_{i}$ as the diameters of outer ring and inner ring respectively.

Obviously, the curvature radius determines the depth when ball enters into the raceway, and the curvature radiuses of ball $c_{d r}$, inner ring $c_{d i}$ and outer ring $c_{d o}$ can be obtained respectively by Equations 19, 20 and 21 (Mishra, Samantaray, and Chakraborty (2017)).

$$
\begin{aligned}
& c_{d r}=\frac{1}{2\left(D_{b}-\sqrt{D_{b}^{2}-B^{2}}\right)}, \\
& c_{d i}=\frac{1}{2\left(D_{i}-\sqrt{D_{i}^{2}-B^{2}}\right)}, \\
& c_{d o}=\frac{1}{2\left(D_{o}-\sqrt{D_{o}^{2}-B^{2}}\right)},
\end{aligned}
$$

During one revolution of the ball, defect contacts the inner ring and outer ring in succession, with an angular distance of $\pi$. So, $c_{d}$ can be given by Equation 22 (Mishra, Samantaray, and Chakraborty (2017)).

$$
c_{d}=\left\{\begin{array}{lr}
c_{d r}-c_{d o}, & 0 \leq \varphi_{s} \leq \Delta \phi_{b o} \\
c_{d r}+c_{d i}, & \pi \leq \varphi_{s} \leq \pi+\Delta \phi_{b i}
\end{array}\right.
$$

Deformation released by fault only appears on the fault ball ( $k^{\text {th }}$ ball). As a result, the contact deformation with ball defect is given by:

$$
\delta_{j}= \begin{cases}0, & j \neq k \\ c_{d}, & j=k\end{cases}
$$

\subsubsection{Multiple Defects}

When bearing has multiple defects, the model is expanded into a matrix. For simplicity, no matter how many and what kind of defects the bearing has, the total impact on the bearing vibration is supposed to be the sum of effect that each defect has on each ball. Thus, a $3 \times n_{d}$ matrix $N$ is defined to deal with multiple defects modeling.

$$
N_{3 \times n_{d}}=\left[\begin{array}{lrlr}
p_{1} & p_{2} & \ldots & p_{n_{d}} \\
\phi_{d_{1}} & \phi_{d_{2}} & \ldots & \phi_{d_{n d}} \\
\Delta \phi_{d_{1}} & \Delta \phi_{d_{1}} & \cdots & \Delta \phi_{d_{n d}}
\end{array}\right]
$$

where $p_{n_{d}}, \phi_{d_{n_{d}}}$ and $\Delta \phi_{d_{n_{d}}}$ stand for the $n_{d}^{\text {th }}$ defect position, defect initial angle and defect span angle respectively. In this model, $p_{i}$ is defined with Equation 25 for 
$i=1,2 \cdots n_{d}$

$$
p_{i}=\left\{\begin{array}{lr}
1, & \text { outer ring fault } \\
2, & \text { inner ring fault } \\
3, & \text { ball fault }
\end{array}\right.
$$

The deformation on each ball caused by each defect can be determined based on above discussion. For a bearing with $n_{b}$ rolling elements and $n_{d}$ defects, the deformation depth is an $n_{d} \times n_{b}$ matrix as:

$$
\delta_{n_{d} \times n_{b}}=\left[\begin{array}{rcc}
\delta_{11} & \cdots & \delta_{1 n_{b}} \\
\vdots & \ddots & \vdots \\
\delta_{n d_{1}} & \cdots & \delta n_{d} n_{b}
\end{array}\right]
$$

The total deformation depth caused by all defects for each ball is obtained through elementwise addition in column. Therefore, a deformation vector with $n_{b}$ dimensions is given as Equation 27.

$$
\delta_{1 \times n_{b}}=\left[\delta_{1}, \delta_{1}, \cdots, \delta_{n_{b}}\right]
$$

Consequently, the defect model for bearing with $n_{d}$ defects is established. The calculation of contact force and acceleration is the same as the single defect model.

\subsubsection{Defect Shape and Size}

Besides defect position, defect shape also has much influence on bearing acceleration response. Most existing bearing defect models, however, simplify the defect depth $c_{d}$ as a constant value. In practice, the value of $c_{d}$ differs with defect shape as well as the ratio of defect size to ball diameter (Cui, X. Chen, and S. Chen (2015)). In order to accurately model the defect shape, the released deformation is modeled by a piece-wise function. In this research, two ratios, ball to defect ratio $\eta_{b d}$ and length to width ratio $\eta_{d}$, are defined in Equation 28 and 29 for defect shape modeling,

$$
\begin{gathered}
\eta_{b d}=\frac{D_{b}}{\min (L, B)}, \\
\eta_{d}=\frac{L}{B},
\end{gathered}
$$

where $B$ and $L$ represent the width and length of the defect respectively (Cui, X. Chen, and S. Chen (2015)). With the combination of these two ratios, the defect shape is grouped into four types $\left(H_{r 1}, H_{r 2}, H_{r 3}, H_{r 4}\right)$, which is given by Equation 30 (Cui, X. Chen, and S. Chen (2015)).

$$
c_{d}=\left\{\begin{array}{lr}
H_{r 1}, & \eta_{b d} \gg 1 \\
H_{r 2}, & \eta_{b d}>1 \text { and } \eta_{d} \leq 1 \\
H_{r 3}, & \eta_{b d}>1 \text { and } \eta_{d}>1 \\
H_{r 4}, & \eta_{b d} \leq 1
\end{array}\right.
$$

The maximal depth of defect for each case is denoted with $c_{d}^{\prime}$, which will be explained later.
In case 1 , the defect size is too small compared with ball diameter, so the ball leaves defect immediately as soon as it contacts the defect. Therefore, as shown in Figure 3(a), there is no deformation change in the defect zone, and $H_{r 1}$ can be modeled as:

$$
H_{r 1}=c_{d}^{\prime}, \quad 0 \leq \varphi_{j} \leq \Delta \phi_{d}
$$

For case 2, the ball's moving path over the defect zone is like a half-sine wave, as shown in Figure 3(b). The deformation released from defect increases gradually to the maximum and then decreases, which can be given by:

$$
H_{r 2}=c_{d}^{\prime} \sin \left(\frac{\pi}{\Delta \phi_{d}} \varphi_{j}\right), \quad 0 \leq \varphi_{j} \leq \Delta \phi_{d}
$$

In case 3, as revealed in Figure 3(c), $c_{d}$ rises up to the maximal depth gradually and remains at the maximum between $\phi_{1}$ and $\phi_{2}$. After that, $c_{d}$ begins to decrease when the ball gets out of defect. This shape is expressed by Equations 33 and 34.

$$
\begin{gathered}
H_{r 3}=\left\{\begin{array}{lr}
c_{d}^{\prime} \sin \left(\frac{\pi}{2 \phi_{1}} \varphi_{j}\right) & 0 \leq \varphi_{j}<\phi_{1} \\
c_{d}^{\prime} & \phi_{1} \leq \varphi_{j}<\phi_{2} \\
c_{d}^{\prime} \sin \left(\frac{\pi}{2 \phi_{1}} \varphi_{j}+\frac{\pi}{2}\right) & \phi_{2} \leq \varphi_{j}<\Delta \phi_{d}
\end{array}\right. \\
\phi_{1}=\Delta \phi_{d} \lambda, \quad \phi_{2}=\Delta \phi_{d}(1-\lambda),
\end{gathered}
$$

hereby $\lambda$ is the ratio of $\phi_{1}$ to $\Delta \phi_{d}$. $\phi_{1}$ is the position where the ball reaches defect bottom.

In case 4 , since the ratio of ball to defect $\eta_{b d}$ is less than that in case 3 . Thus, the ball reaches the defect bottom within a complete 1/4 sine wave, and defect shape can be modeled as:

$$
H_{r 4}=\left\{\begin{array}{lc}
c_{d}^{\prime} \sin \left(\frac{2 \pi}{\Delta \phi_{d}} \varphi_{j}\right), & 0 \leq \varphi_{j}<\phi_{1} \\
c_{d}^{\prime}, & \phi_{1} \leq \varphi_{j}<\phi_{2} \\
c_{d}^{\prime} \sin \left(2 \pi-\frac{2 \pi}{\Delta \Phi_{d}} \varphi_{j}\right), & \phi_{2} \leq \varphi_{j}<\Delta \phi_{d}
\end{array}\right.
$$

The maximum depth $c_{d}^{\prime}$ is given by Equations (Cui, $\mathrm{X}$. Chen, and S. Chen (2015)):

$$
H_{d}=\frac{D_{b}}{2}-\sqrt{\left(\frac{D_{b}}{2}\right)^{2}-\left(\frac{B}{2}\right)^{2}},
$$

with

$$
c_{d}^{\prime}=\min \left(H, H_{d}\right)
$$




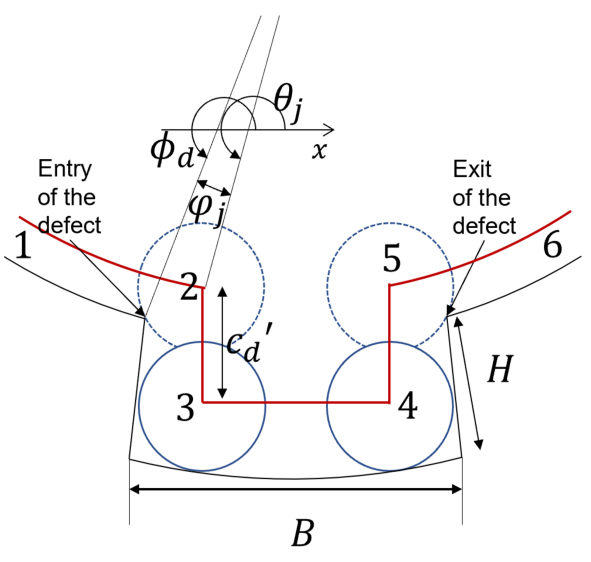

(a) $H_{r 1}$

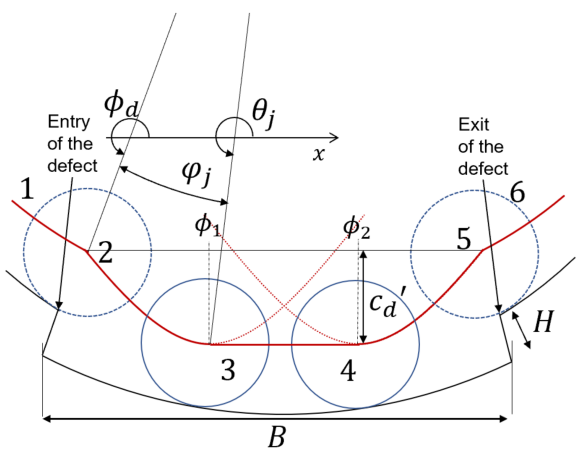

(c) $H_{r 3}$

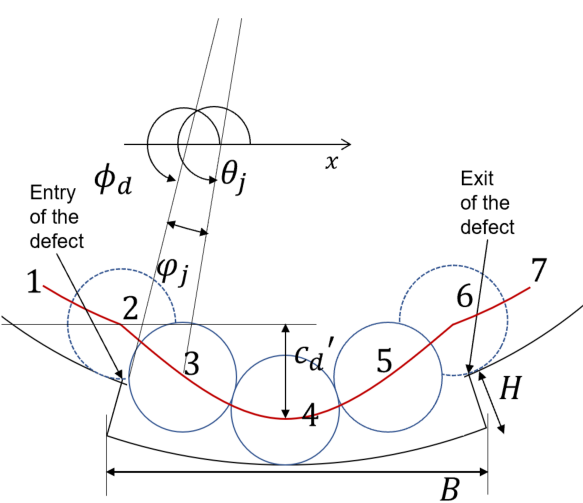

(b) $H_{r 2}$

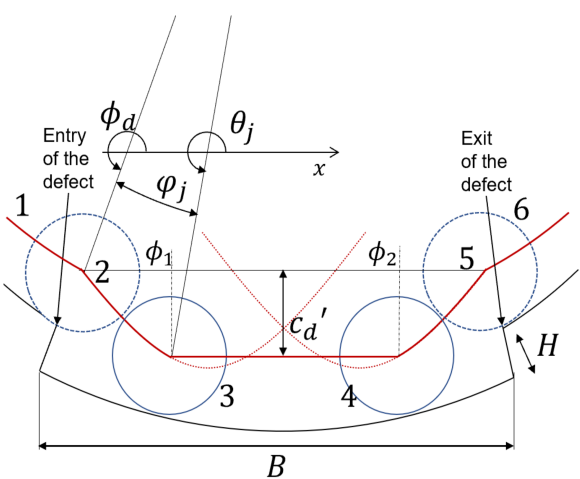

(d) $H_{r 4}$

Figure 3. Defect depth $c_{d}$ under different defect shape.

Table 1. Bearing parameters.

\begin{tabular}{llr}
\hline Symbol & Quantity & Value \\
\hline$m_{s}$ & Shaft mass & $3,2638 \mathrm{~kg}$ \\
$R_{s}$ & Shaft damping & $1,3768 \cdot 10^{3} \mathrm{~N} \mathrm{~s} \mathrm{~m}^{-1}$ \\
$K_{s}$ & Shaft stiffness & $7,42 \cdot 10^{7} \mathrm{~N} \mathrm{~m}^{-1}$ \\
$m_{p}$ & Pedal mass & $6,638 \mathrm{~kg}$ \\
$R_{p}$ & Pedal damping & $2,2107 \cdot 10^{3} \mathrm{~N} \mathrm{~s} \mathrm{~m}^{-1}$ \\
$K_{p}$ & Pedal stiffness & $1,51 \cdot 10^{7} \mathrm{~N} \mathrm{~m}^{-1}$ \\
$m_{R}$ & Resonator mass & $1 \mathrm{~kg}$ \\
$R_{R}$ & Resonator stiffness & $9,4248 \cdot 10^{3} \mathrm{~N} \mathrm{~s} \mathrm{~m}^{-1}$ \\
$K_{R}$ & Resonator stiffness & $8,8826 \cdot 10^{9} \mathrm{~N} \mathrm{~m}^{-1}$ \\
$n_{b}$ & Ball number & 9 \\
$D_{p}$ & Pitch diameter & $3,932 \cdot 10^{-2} \mathrm{~m}$ \\
$D_{b}$ & Ball diameter & $7,94 \cdot 10^{-3} \mathrm{~m}$ \\
$\phi_{\text {slip }}$ & Ball slip angle & $0,01 \mathrm{rad}^{-1}$ \\
$r a n d$ & Mutation percentage & 0 \\
$K_{b}$ & Ball stiffness & $1,89 \cdot 10^{10} \mathrm{Nm}^{-1}$ \\
$c$ & Bearing clearance & 0 \\
$\alpha$ & Contact angle & $0^{\circ}$ \\
\hline
\end{tabular}

Table 2. Defect parameters.

\begin{tabular}{llr}
\hline Symbol & Quantity & Value \\
\hline$L$ & Axial defect length & $3 \cdot 10^{-4} \mathrm{~m}$ \\
$B$ & Race defect width & $10 \cdot 10^{-4} \mathrm{~m}$ \\
$H$ & Radial defect depth & $6 \cdot 10^{-4} \mathrm{~m}$ \\
$\lambda$ & Ratio of $\phi_{1}$ to $\Delta \phi_{d}$ & 0,2 \\
$\phi_{d}$ & Initial defect position & $270^{\circ}$ \\
$k$ & Order of the defective ball & 4 \\
$w$ & Spall width & $3 \cdot 10^{-3} \mathrm{~m}$ \\
$\phi_{s_{i n i}}$ & Initial position of spall & $0^{\circ}$ \\
\hline
\end{tabular}

\subsubsection{Parameters}

Parameter specification of the 5-DoF dynamics model is shown in Table 1, which includes geometrical and material parameters (Mishra, Samantaray, and Chakraborty (2017)). The defect model parameters can be defined by users out of simulation requirements. In this paper, they are set as in Table 2 .

Once the bearing model has been finished, the characteristic frequencies can be calculated by Equations 38-42, 
including ball pass frequency of outer ring $(B P F O)$, ball pass frequency of inner ring $(B P F I)$, ball spin frequency $(B S F)$, fundamental train frequency $(F T F)$ and element defect frequency $(E D F)$. All of them will be used for analysis and discussion in the following sections (Robert B Randall and Antoni (2011)).

$$
\begin{gathered}
B P F O=\frac{n_{b} f}{2}\left(1-\frac{D_{b}}{D_{p}} \cos \alpha\right) \\
B P F I=\frac{n_{b} f}{2}\left(1+\frac{D_{b}}{D_{p}} \cos \alpha\right) \\
B S F=\frac{f D_{p}}{2 D_{b}}\left[1-\left(\frac{D_{b}}{D_{p}} \cos \alpha\right)^{2}\right] \\
F T F=\frac{f}{2}\left(1-\frac{D_{b}}{D_{p}} \cos \alpha\right) \\
E D F=2 B S F
\end{gathered}
$$

\subsection{Driving System}

Besides test bearing, the virtual bearing test bench also consists of a driving module and a loading module, where the loading module guarantees that test bearing works under defined external load, and driving module is responsible for speed profile definition. In this paper, the driving module is modeled by a DC motor and a shaft, while the loading module is modeled by an electro-hydraulic servo system.

Based on Newton's second law and Kirchhoff's voltage law, the DC motor can be modeled as:

$$
\begin{gathered}
J_{M} \frac{d \omega}{d t}+C_{n} \omega+T_{L}=T_{e}, \\
L_{M} \frac{d i}{d t}+R i+e=U,
\end{gathered}
$$

where the generated torque $T_{e}$ and the back electromotive force $(\mathrm{EMF}) e$ can be further modeled as following.

$$
\begin{aligned}
& T_{e}=K_{t} \cdot i, \\
& e=K_{e} \cdot \omega .
\end{aligned}
$$

The speed of the DC motor is controlled by a PID controller.

\subsection{Shaft}

The connecting shaft is employed to transmit the moment $T_{e}$ from DC-motor. Its dynamics is modeled as:

$$
k_{S} \int\left(\omega_{\text {in }}-\omega_{\text {out }}\right) d t+c_{S}\left(\omega_{\text {in }}-\omega_{\text {out }}\right)=T_{e}-T_{L},
$$

where $k_{S}$ is the stiffness and $c_{S}$ is the damping coefficient, $\omega_{\text {in }}$ and $\omega_{\text {out }}$ are the input and output speed of the shaft, $T_{L}$ is load torque.

\subsection{Loading System}

\subsubsection{Components of Electro-hydraulic Servo System}

The electro-hydraulic servo system consists of three components: servo amplifier, servo valve and actuator. The servo amplifier is used to convert signal from voltage to current, the servo valve is a proportional relief valve, and the actuator is a hydraulic cylinder. The asymmetrical cylinder is controlled by a four-way valve with position feedback.

\subsubsection{Modeling of Electro-hydraulic Servo System}

The servo amplifier is modeled as a proportional component, which amplifies the control voltage and then converts into current $i$ to control the electromagnetic force acting on the valve spool,

$$
i(t)=K_{f} u_{e}(t)
$$

The servo valve is modeled as a second-order system like follows (Rydberg (2016)).

$$
G_{s v}(s)=\frac{Q_{L}(s)}{I(s)}=\frac{K_{g y}}{\frac{s^{2}}{\omega_{s v}^{2}}+\frac{2 \xi_{s v}}{\omega_{s v}} s+1} .
$$

The gain is obtained from

$$
K_{s v}=\frac{K_{I E}}{K_{S F}} \cdot K_{Q}
$$

where $K_{I F}, K_{S F}$ and $K_{q}$ are the gain of current to force, the stiffness of valve spool and the flow gain respectively. The natural frequency and servo valve damping coefficient are given by:

$$
\begin{aligned}
& \omega_{s v}=\sqrt{K_{s F} / m}, \\
& \xi_{s v}=\frac{B_{p}}{2 \sqrt{m K_{S F}}} .
\end{aligned}
$$

The actuator in this study is modeled as an asymmetric cylinder, which can be regarded as a valve-controlled piston with position feedback. In general, three sub-models are developed to describe the flow characteristics, flow balance and force balance respectively. The flow characteristics after linearization can be simplified as:

$$
Q_{L}(s)=K_{q} X_{v}(s)-K_{c} P_{L}(s)
$$

the flow balance equation is identified as:

$$
Q_{L}(s)=A_{p} s X_{p}(s)+\lambda_{c} P_{L}(s)+\frac{v_{t}}{4 \beta_{e}} s P_{L}(s),
$$

and the force balance equation can be formulated as:

$$
A_{p} P_{L}(s)=M_{t} s^{2} X_{p}(s)+B_{c} X_{p}(s)+F_{L},
$$

where $X_{v}$ and $K_{c}$ are the displacement of valve and the flow-pressure factor. $Q_{L}, X_{p}$ and $P_{L}$ stand for the load flow, the displacement of piston and the pressure of the load 


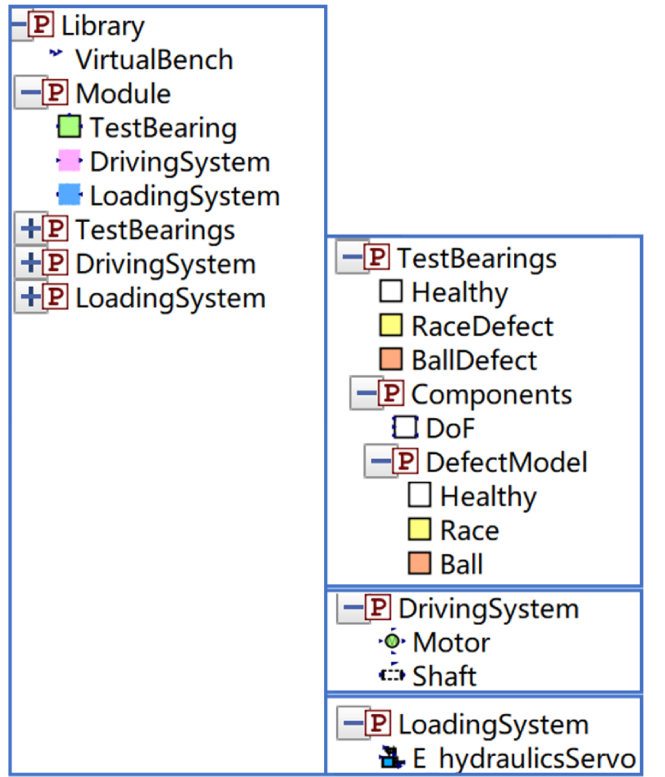

Figure 4. Structure of the developed library in OpenModelica.

flow respectively. $A_{p}, \lambda_{c}, V_{t}$ and $\beta_{e}$ are the piston area, the total leakage coefficient, the cylinder volume as well as the volume elastic modulus coefficient. $M_{t}$ and $B_{c}$ are the mass of piston and the damping factor of cylinder.

Combining Equations 53 to 55 gives the transfer function of piston displacement to valve displacement, as shown in Equation 56, where the total leakage $\lambda_{c}$ is omitted and the damping factor is assumed to be small (Mitianiec and Bac (2011)).

$$
G_{h}(s)=\frac{X_{p}(s)}{X_{v}(s)}=\frac{K_{q} / A_{p}}{s\left(\frac{s^{2}}{\omega_{h}^{2}}+\frac{2 \xi_{h}}{\omega_{h}} s+1\right)},
$$

with

$$
\begin{gathered}
\omega_{h}=\sqrt{\frac{4 \beta_{e} A_{\vec{p}}^{2}}{V_{t} M_{t}}}, \\
\xi_{h}=\frac{K_{c}}{A_{p}} \sqrt{\frac{\beta_{e} M_{\mathrm{t}}}{V_{t}}} .
\end{gathered}
$$

In short, a proportional system is used to model the amplifier, $G_{s v}$ for the servo valve and $G_{h}$ for hydraulic cylinder. The loading system is also controlled by a PID controller.

\section{Implementation}

In this research, a model library is created for a virtual bearing test bench in OpenModelica-v1.16.0. As shown in Figure 4(left), 3 main modules like TestBearings, DrivingSystem and LoadingSystem are packaged and can be used as plug-in components in modeling. Figure 4(right) displays the components used in each module, and they are also sub-packaged with corresponding names. Like the TestBearings package provides three instance models as Healthy, RaceDefect and BallDefect and a Com-

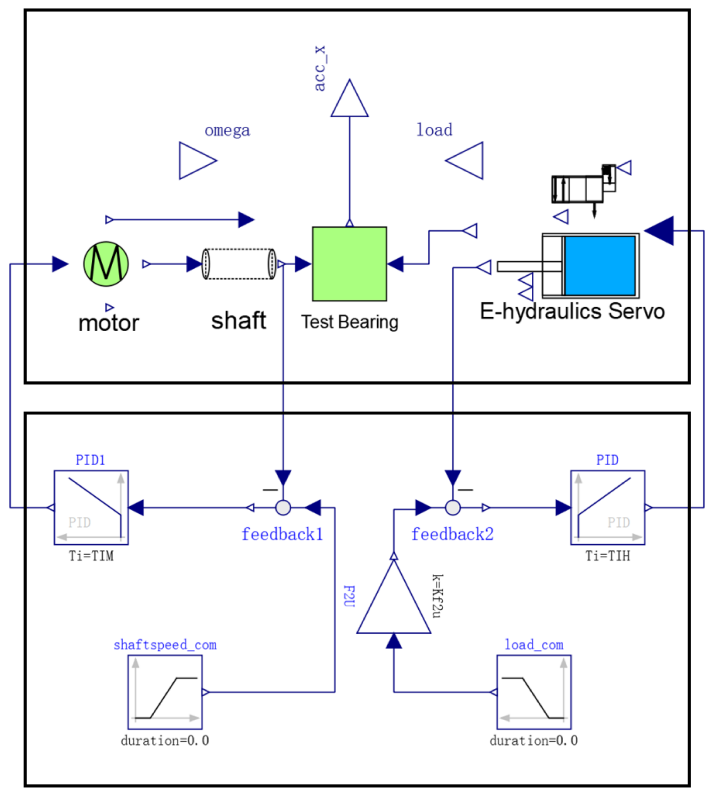

Figure 5. Layer diagram of the VirtualBench.

ponents sub-package containing a DoF model and another sub-package named DefectModel.

These models can be constructed into any configuration as required, all components, sub-packages and models can be used separately or in combination to meet user demand. Nevertheless, a configuration instance, VirtualBench, is provided at the top of Library.

\subsection{System Configuration}

Figure 5 demonstrates the layer diagram of proposed test bench, which consists of physical part (top) and controller part (bottom). The physical part is established with models developed in above sections, and it outputs three signals, namely rotational speed "omega", acceleration " $a c c_{x}$ " and radial load "load". Moreover, the rotational speed and radial load are inputs to the controller part to provide required conditions for test bearing. The TestBearing model has all parameters mentioned in Section 2.1, with four pages of parameter-input dialog box for users to define fault position, design parameters, material parameters, and defect parameters. Specifically, "position" is a selection parameter to define where the defect is located; design parameters include basic geometrical information such as ball number, pitch diameter and ball diameter; material properties include mass, stiffness, and damping factor of the outer ring, inner ring, rolling element as well as the resonator. Besides, the most important parameters are defect properties. Different parameters are required for different defect scenarios. As long as the number of defects and other defect parameters are given, this defect model can be used for multiple defects as well. The operating conditions are provided by Motor, Shaft, and E_hydraulicsServo. Generally, the TestBearing model can be employed to study the vibration response of fault bearing and all the parameters of any model or com- 


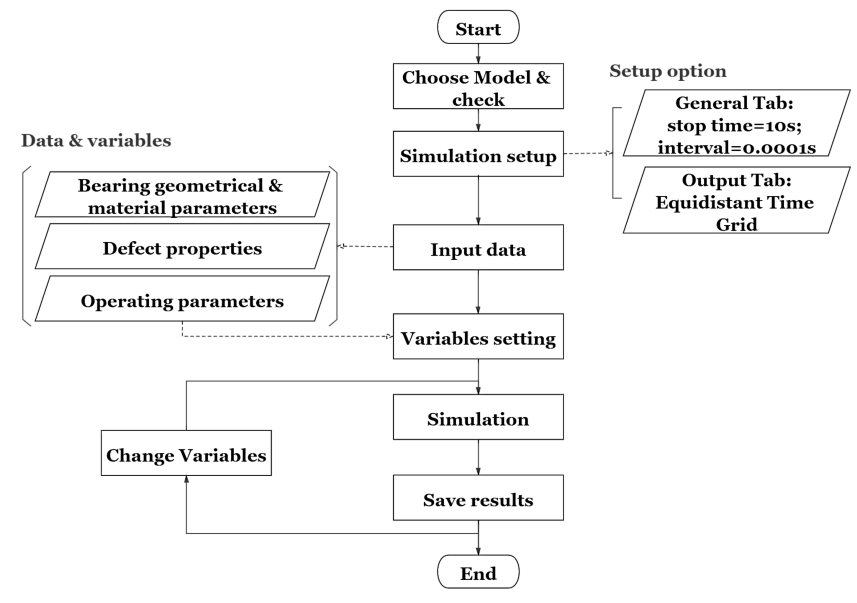

Figure 6. Flowchart of simulation with the virtual bearing test bench.

ponent can be defined by users for specific objectives.

\subsection{Procedure of Virtual Bearing Test Bench}

The flowchart in Figure 6 demonstrates the process to run simulations with proposed virtual bearing test bench. Firstly, the model configuration and precheck is required, specific simulation model should be constructed based on the developed Modelica components. After that, it is necessary to set simulation period, interval length and the output format. Then, in the step of Input data, geometrical and material parameters, defect properties and operating conditions should be defined, and some parameters can be selected as variables to study the effects of defects on vibration from different aspects. At last, run the simulation and save results.

\subsection{Case Simulation and Analysis}

Based on the developed bearing test bench, three simulation cases are conducted for validation. Case $\mathrm{A}$ focuses on the defect position, case $\mathrm{B}$ and case $\mathrm{C}$ deals with multiple defects and defect shape respectively. Simulation time and interval length of 3 cases are set as $10 \mathrm{~s}$ and $0.0001 \mathrm{~s}$, with "DASSL" solver as the integration method.

\subsubsection{Case A: Defect Position}

In case $\mathrm{A}$, three simulations are designed to obtain the bearing responses when a defect occurs on the outer ring, inner ring or a ball respectively. The signal characteristics in both time-domain and frequency-domain are analyzed.

Figure 7 shows the time domain response and envelope spectrum of bearing with a single defect on the outer ring. The theoretical fault characteristic frequency $(B P F O)$ is $35.91 \mathrm{~Hz}$, corresponding to $0.0278 \mathrm{~s}$. In time domain, the impulse decaying oscillation repeats with a period of $0.0279 \mathrm{~s}$, and the impulse magnitude is nearly constant. In frequency domain, BPFO $(35.94 \mathrm{~Hz})$ is extracted in the envelope spectrum, which is very close to the theoretical value.

Figure 8 describes the simulated signal when a defect is

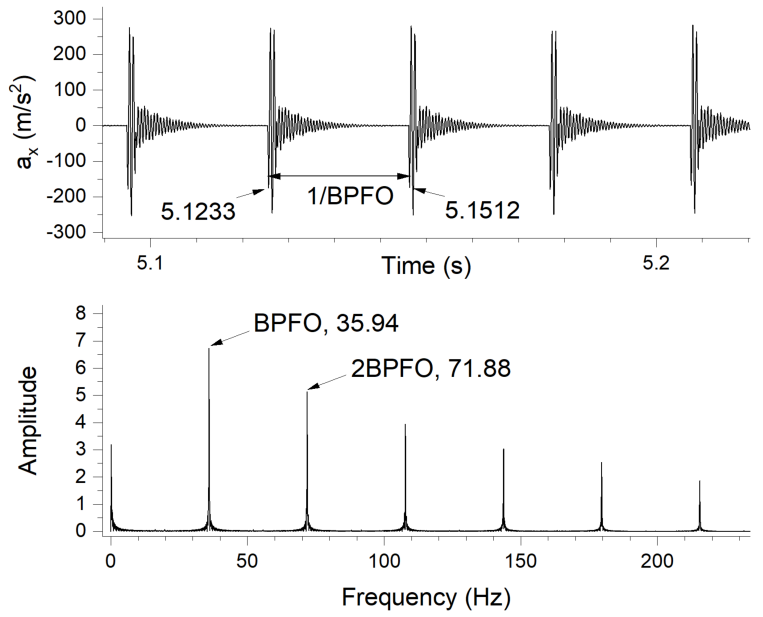

Figure 7. Response in time-domain and envelope spectrum of bearing with outer race fault.

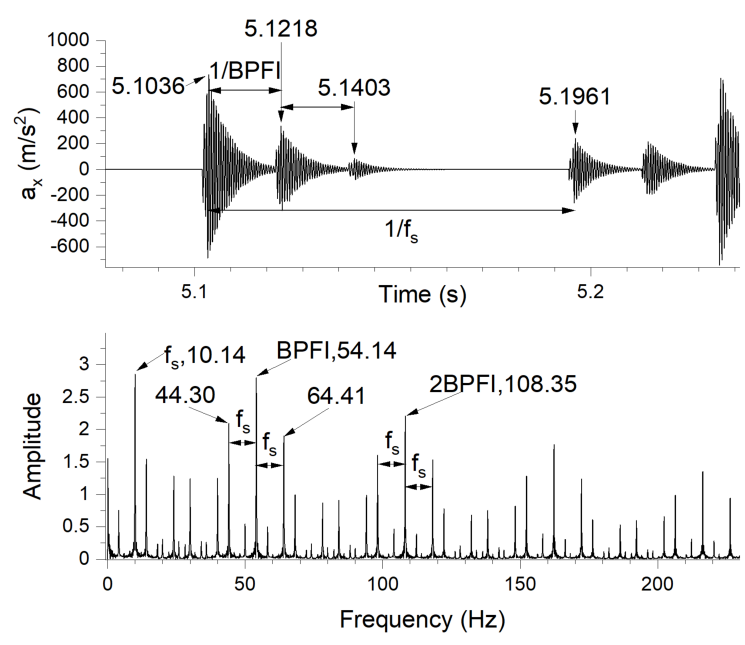

Figure 8. Response in time-domain and envelope spectrum of bearing with inner race fault.

defined on the inner ring. The shaft frequency $\left(f_{s}\right)$ is set as $10 \mathrm{~Hz}$, so the theoretical $B P F I$ is $54.09 \mathrm{~Hz}(0.018 \mathrm{~s})$. The vibration response from $5.1036 \mathrm{~s}$ to $5.1961 \mathrm{~s}$ represents the output during a whole revolution of inner ring, with $5.1036 \mathrm{~s}-5.1403 \mathrm{~s}$ standing for the load zone and $5.1403 \mathrm{~s}-5.1961 \mathrm{~s}$ the non-load zone. The time interval between $5.1036 \mathrm{~s}$ and $5.1961 \mathrm{~s}$ is $0.0925 \mathrm{~s}$, which corresponds to $f_{s}(10.14 \mathrm{~Hz})$ in the envelope spectrum. Within one cycle, there are three peaks at $5.1036 \mathrm{~s}, 5.1218 \mathrm{~s}$ and $5.1403 \mathrm{~s}$, and every two adjacent peaks are $0.018 \mathrm{~s}$ apart away, which is related to the BPFI. Furthermore, the inner ring defect rotates with time, which results in load change at the defect position. Thus, the signal presents various amplitudes during one cycle.

The vibration response of bearing with a defect on the ball is demonstrated in Figure 9. In time-domain, the time interval between every two impulses is approximate $0.021 \mathrm{~s}$. When a defect occurs on a ball, the defect strikes both the outer ring and inner ring in a full rotation. As a result, peaks can be found in frequency-domain at $E D F$ and its 


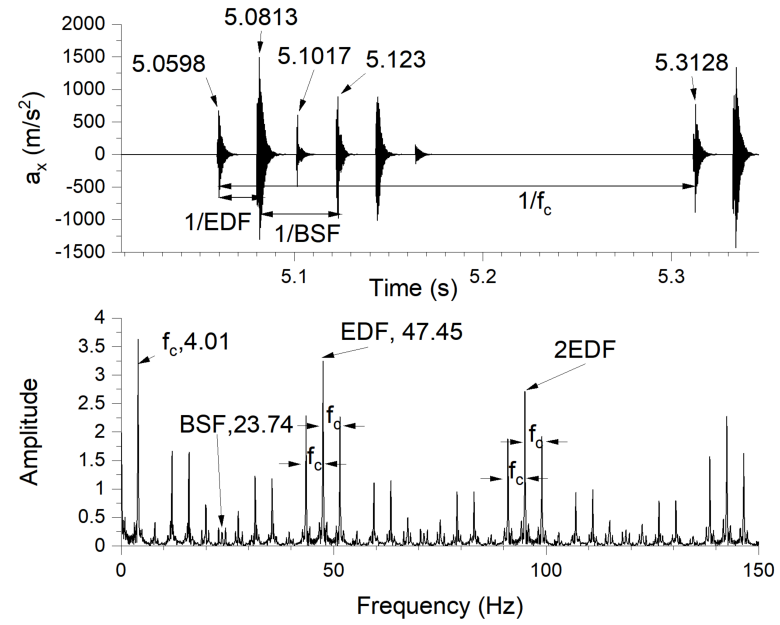

Figure 9. Response in time-domain and envelope spectrum of bearing with ball fault.

harmonics, with $f_{c}$ as the sideband. The theoretical EDF is $47.50 \mathrm{~Hz}$, and the simulated value is $47.45 \mathrm{~Hz}$.

In short, both time-domain and frequency-domain responses contain useful defect information. In timedomain, defect position (outer ring, inner ring or ball) can be deduced from the time interval between adjacent peaks. In frequency-domain, defect position can be inferred from the characteristic frequencies $(B P F O, B P F I$ or $E D F)$ in the envelope spectrum. Furthermore, the amplitude of peaks under different fault positions varies accordingly. The peak amplitudes are nearly constant when a defect occurs on the outer ring, however, the peak amplitudes change during a cycle when a defect happens on the inner ring or a ball. In addition, in the inner-ring defect, $f_{s}$ can be found in the envelope spectrum as sideband, while in the ball defect, the sideband is replaced by $f_{c}$.

\subsubsection{Case B: Multiple Defects}

The second case focuses on multiple defects. The angle between two adjacent defects is defined as $\psi$ and the angle between every two rolling elements in this study is $40^{\circ}$. Therefore, in total, there are 3 relations: $\psi>40^{\circ}, \psi<$ $40^{\circ}, \psi=40^{\circ}$. Given space limitation, only the case with 2 defects and $\psi<40^{\circ}$ is simulated.

Two defects are defined at $255^{\circ}$ and $285^{\circ}$. Once the rolling elements rotate, each ball collides with these two defects successively, resulting in two sequences of collisions. Therefore, in Figure 10, two impacts are observed in a cycle, which identifies the number of defects. According to the direction of acceleration, the impacts at $5.0712 \mathrm{~s}$ (B) and $5.0990 \mathrm{~s}(\mathrm{D})$ are caused by the defect at $255^{\circ}$, while the impacts at $5.0642 \mathrm{~s}(\mathrm{~A})$ and $5.0920 \mathrm{~s}(\mathrm{C})$ by defect at $285^{\circ}$.

The time delay between two strikes due to multiple defects on the races can be calculated as follows (Patel, Tan-

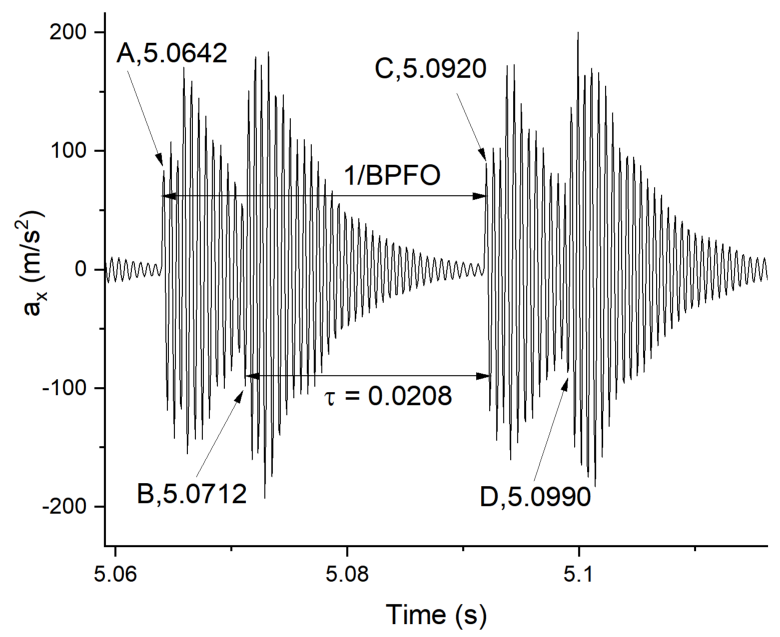

Figure 10. Bearing response with two defects on the outer ring separated by $30^{\circ}$.

Table 3. Time delay of two defects.

\begin{tabular}{ll}
\hline$\Phi$ & $30^{\circ}$ \\
\hline$\tau$ calculated [s] & 0.0209 \\
$\tau$ simulated [s] & 0.0208 \\
\hline
\end{tabular}

don, and Pandey (2014)).

$$
\tau(\Phi)= \begin{cases}\frac{\Phi}{x f_{t}}, & x \leq \Phi \\ \frac{a b s(x-\Phi)}{x f_{t}}, & x>\Phi\end{cases}
$$

with

$$
f_{t}= \begin{cases}B P F O, & \text { defects on outer ring } \\ B P F I, & \text { defects on inner ring }\end{cases}
$$

The time delay between B and C is $0.0208 \mathrm{~s}$, which corresponds to the angle between $255^{\circ}$ and $285^{\circ}$. The theoretical time delay and simulation result are summarized in Table 3.

\subsubsection{Case C: Defect Shape}

Case $\mathrm{C}$ is designed to study the relationship between defect shape and vibration response, with a rectangle defect defined for validation. The defect is located at $270^{\circ}$, the width and length are defined as $1.5 \times 10^{-4} \mathrm{~m}$ and $3 \times 10^{-4} \mathrm{~m}$. With shaft frequency set as $1 \mathrm{~Hz}$ and radial load as $-30 \mathrm{kN}$, the vibration signal is presented in Figure 11.

There are three peaks in a cycle, which appears at $4.5313 \mathrm{~s}, 4.6712 \mathrm{~s}$ and $4.8103 \mathrm{~s}$ respectively, as shown in Figure 11. These 3 peaks represent the time points when a ball enters and leaves the load zone, and then enters into the load zone again, respectively. Only the balls in load zone generate deformations, so the acceleration changes suddenly at the entry and exit of load zone. Therefore, acceleration between $4.5313 \mathrm{~s}$ and $4.6712 \mathrm{~s}$ in Figure 11 is the signal that occurs in defect zone. 


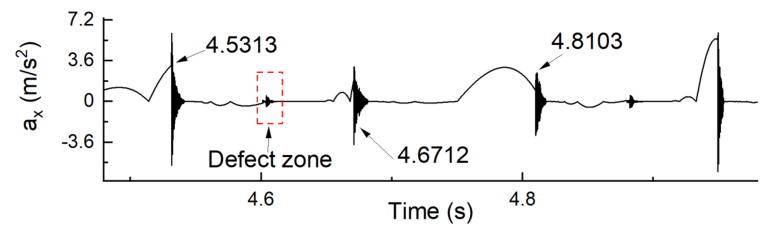

Figure 11. Acceleration in time-domain of bearing with a rectangle-shape defect.

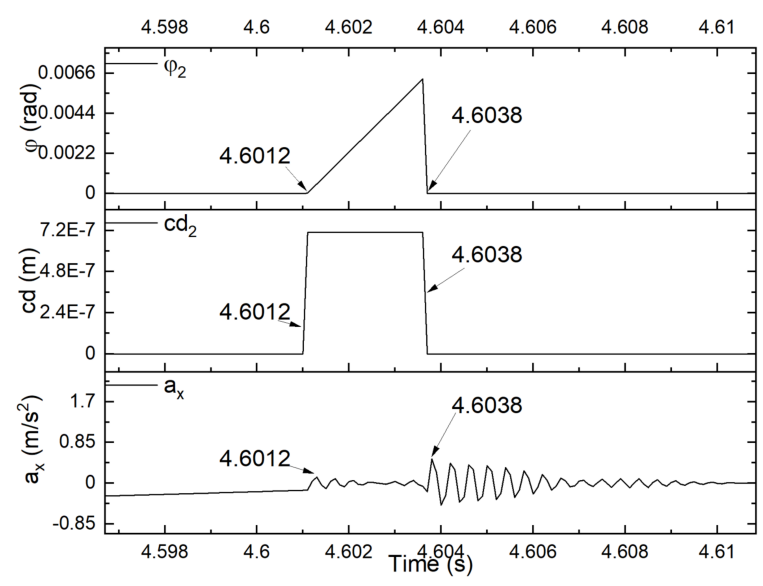

Figure 12. Signal output with a rectangle-shape defect: $\phi_{2}$ : angle between ball and defect start edge; $c d_{2}$ : additional deformation of ball caused by defect; $a_{x}$ : acceleration in x-direction.

To further study the signal in defect zone, the angle between ball center and the defect starting edge $\left(\phi_{2}\right)$, and the additional deformation generated by the defect $\left(c d_{2}\right)$ are presented to demonstrate the transient process when the 2nd ball passes through the defect zone. As shown in Figure $12, \phi_{2}$ and $c d_{2}$ increase at $4.6012 \mathrm{~s}$, indicating that the ball enters into the defect zone at this time. Thus, $a_{x}$ shows an impulse at this moment. Likewise, the peak at $4.6038 \mathrm{~s}$ is the result of ball exiting because $\phi_{2}$ changes to 0 at this point. The change of $c d_{2}$ presents a rectangle profile, which agrees well with the defined defect shape.

\section{Conclusion}

In this paper, a model of the whole bearing test bench including test bearing, connecting shaft, driving system and loading system is developed in OpenModelica. The proposed virtual test bench can be used to simulate bearing dynamics response, especially under different defect scenarios characterized by defect position, multiple defects, defect shape and defect size. It can be also employed as an alternative to a real test bench to generate fault signals for fault diagnosis algorithm development and validation, which could be a good supplement of experimental measurement when a large amount of data is required in machine learning or deep learning methods. The modeling theory and implementation process of the whole best bench are detailed, and three cases are designed to validate its effectiveness.

Due to the advantages in characteristics of open source, the OpenModelica has much superiority over the MATLAB/Simulink, furthermore, it also has more user-friendly interfaces with Python. In the future, the virtual bearing test bench developed in this paper will be adopted to study the transfer learning from the physics model to the real test bench.

\section{Acknowledgements}

This work is supported by CSC doctoral scholarship 201806250024 and Zhejiang Lab's International Talent Fund for Young Professionals.

\section{References}

Cong, Feiyun et al. (2013). "Vibration model of rolling element bearings in a rotor-bearing system for fault diagnosis". In: Journal of sound and vibration 332.8, pp. 2081-2097.

Cui, Lingli, Xue Chen, and Shujun Chen (2015). "Dynamics modeling and analysis of local fault of rolling element bearing”. In: Advances in Mechanical Engineering 7.1, p. 262351.

Ho, D and RB Randall (2000). "Optimisation of bearing diagnostic techniques using simulated and actual bearing fault signals". In: Mechanical systems and signal processing 14.5, pp. 763-788.

Liu, Jing, Yimin Shao, and Teik C Lim (2012). "Vibration analysis of ball bearings with a localized defect applying piecewise response function". In: Mechanism and Machine Theory 56, pp. 156-169.

McFadden, PD and JD Smith (1984). "Model for the vibration produced by a single point defect in a rolling element bearing”. In: Journal of sound and vibration 96.1, pp. 69-82.

Mishra, C, AK Samantaray, and G Chakraborty (2017). "Ball bearing defect models: A study of simulated and experimental fault signatures". In: Journal of Sound and Vibration 400, pp. 86-112.

Mitianiec, Wladyslaw and Jarosław Bac (2011). "Mathematical model of the hydraulic valve timing system". In: Journal of KONES 18, pp. 311-321.

Patel, VN, N Tandon, and RK Pandey (2014). "Vibrations generated by rolling element bearings having multiple local defects on races”. In: Procedia Technology 14, pp. 312-319.

Randall, Robert B and Jerome Antoni (2011). "Rolling element bearing diagnostics-A tutorial". In: Mechanical systems and signal processing 25.2, pp. 485-520.

Rydberg, Karl-Erik (2016). Hydraulic Servo Systems: Dynamic Properties and Control. 